\title{
Defining winning strategies in fixed-point logic
}

\author{
Felix Canavoi ${ }^{\dagger}$, Erich Grädel*, Simon Leßenich* and Wied Pakusa* \\ * RWTH Aachen University, \{graedel,lessenich,pakusa\}@logic.rwth-aachen.de \\ $\dagger$ TU Darmstadt, canavoi@mathematik.tu-darmstadt.de
}

\begin{abstract}
We study definability questions for positional winning strategies in infinite games on graphs.

The quest for efficient algorithmic constructions of winning regions and winning strategies in infinite games, in particular parity games, is of importance in many branches of logic and computer science. A closely related, yet different, facet of this problem concerns the definability of winning regions and winning strategies in logical systems such as monadic second-order logic, least fixed-point logic LFP, the modal $\mu$-calculus and some of its fragments. While a number of results concerning definability issues for winning regions have been established, so far almost nothing has been known concerning the definability of winning strategies.

We make the notion of logical definability of positional winning strategies precise and study systematically the possibility of translations between definitions of winning regions and definitions of winning strategies. We present explicit LFP-definitions for winning strategies in games with relatively simple objectives, such as safety, reachability, eventual safety (Co-Büchi) and recurrent reachability (Büchi), and then prove, based on the Stage Comparison Theorem, that winning strategies for any class of parity games with a bounded number of priorities are LFP-definable. For parity games with an unbounded number of priorities, LFP-definitions of winning strategies are provably impossible on arbitrary (finite and infinite) game graphs. On finite game graphs however, this definability problem turns out to be equivalent to the fundamental open question about the algorithmic complexity of parity games. Indeed, based on a general argument about LFP-translations we prove that LFPdefinable winning strategies on the class of all finite parity games exist if, and only if, parity games can be solved in polynomial time, despite the fact that LFP is, in general, strictly weaker than polynomial time.
\end{abstract}

\section{INTRODUCTION}

Infinite games on graphs, where two players move a token along the edges of a directed graph tracing out a finite or infinite path, are intimately connected with fundamental questions in logic and have numerous applications in different areas of mathematics and computer science. We consider here games with qualitative objectives: For each player, we have a winning condition, which is either specified by a logical formula on infinite paths (typically from monadic secondorder logic S1S, first-order logic FO, or temporal logic LTL) or formulated as a classical Muller, Streett-Rabin, or parity condition. For such a game $\mathcal{G}$, a position $v$ and a player $\sigma \in\{0,1\}$, the question we ask is whether Player $\sigma$ has a winning strategy in $\mathcal{G}$ from position $v$. To solve a game algorithmically thus means to compute winning regions and winning strategies for the two players, where the winning region of a player is the set of those positions from which she has a winning strategy. While efficient algorithms exist for many classes of games, including those where the players have reachability, safety, recurrent reachability (Büchi) or eventual safety (Co-Büchi) objectives, the question whether the winning regions in parity games can be computed in polynomial time is one of the most important open problems in the field of infinite games. In parity games, one assigns to each position a natural number, called its priority, and the winner of an infinite play depends on whether the least priority occurring infinitely often is even or odd. Parity games are important because many games arising in practical applications, including all games with $\omega$-regular winning conditions, can be reduced to parity games, because parity games arise as the model checking games for fixed-point logics, and finally because parity games always admit positional (i.e. memoryless) winning strategies. As a direct consequence of this fact, it follows that the problem of solving parity games is in NP $\cap$ Co-NP. The best known deterministic algorithm has complexity $n^{O(\sqrt{n})}$ [14]. Much effort has been put into identifying and classifying classes of parity games that guarantee efficient algorithmic solutions. For instance, there are deterministic polynomial-time algorithms for any class of parity games with a bounded number of priorities [13], and for parity games with certain restrictions on the underlying game graph, such as games where even and odd cycles do not intersect, solitaire games and nested solitaire games [3], and parity games of bounded tree width [17], bounded entanglement [4], bounded DAG-width [2], [18], bounded Kelly-width [11], or bounded clique width [19].

Definability of winning regions. A closely related problem concerns the definability of winning regions and winning strategies in logical systems such as monadic second-order logic, least fixed-point logic LFP, the modal $\mu$-calculus and some of its fragments. Such a study of the descriptive complexity of games, i.e. of the logical resources needed for specifying winning regions and winning strategies provides insights into the structure of the associated algorithmic problems, and the sources of their algorithmic difficulty; on the other hand, definability and non-definability results on games also have important applications on the structure and expressive power of logical systems.

Given a logic $L$ and a class $\mathcal{S}$ of games, presented as 
relational structures of some fixed vocabulary $\tau$, we say that winning regions on $\mathcal{S}$ are definable in $L$, if there exist formulae $\psi_{0}(x)$ and $\psi_{1}(x)$ of $L(\tau)$ that define, on each game $\mathcal{G} \in \mathcal{S}$, the winning regions $W_{0}$ and $W_{1}$ for the two players. This means that, for each game $\mathcal{G} \in \mathcal{S}$ and each player $\sigma \in\{0,1\}$,

$$
W_{\sigma}=\left\{v \in \mathcal{G}: \mathcal{G}=\psi_{\sigma}(v)\right\}
$$

It is an obvious consequence of standard facts of finite model theory, such as Gaifman's theorem on the locality of first-order logic (FO), that FO is too weak for games, even for very simple objectives such as reachability and safety. On the other hand, it can be shown that on any class $\mathcal{S}$ of games on which the objectives of the two players can be uniformly described by formulae of S1S (which depend on a bounded vocabulary of monadic predicates), the winning regions for the two players are definable in LFP, in MSO, and also in the modal $\mu$ calculus. This includes games with standard objectives such as reachability, safety, recurrent reachability and eventual safety, and indeed all parity, Streett-Rabin, and even Muller conditions with a bounded number of priorities. Formulae defining winning regions in parity games with priorities $0, \ldots, d-1$, for any fixed $d$, have been essential for settling structural properties of fixed point logics. In the modal $\mu$-calculus $L_{\mu}$ such formulae require $d$ nested fixed points that alternate between least and greatest fixed points, and thus witness the strictness of the alternation hierarchy. It has been shown that such formulae can also be constructed in Parikh's game logic GL and in the two-variable fragment of the $\mu$-calculus [1], [5] which proves that these fragments of $L_{\mu}$ nontrivially intersect all levels of the alternation hierarchy. By a different use of games, also the strictness of the variable hierarchy of the $\mu$-calculus could be settled in [5]. However, definability issues for classes of games where the objectives depend on an unbounded collection of local parameters (colours, priorities, atomic propositions etc.) are quite different. First of all, games in such classes require a somewhat more complicated presentation as relational structures. Parity games, for instance, can be presented as game graphs with a preorder on the positions, where $u \prec v$ means that $u$ has a smaller (i.e. more relevant) priority than $v$. Definability issues of such classes of games have been investigated in [8] and it has been shown that definability results depend on whether only finite game graphs are considered, or also infinite ones. As a consequence of the strictness of the alternation hierarchy for LFP on arithmetic and by means of an interpretation argument for model checking games, it has been shown in [8] that winning regions of parity games are, in general, not LFP-definable (even if we restrict attention to games on a countable graph and with finitely many priorities). On finite game graphs, however, this may well be different. Indeed the winning regions are LFP-definable if, and only if, they are computable in polynomial-time.

Definability of positional winning strategies. In this paper we address the much less understood problem of defining winning strategies rather than just winning regions. Our moti- vation comes from the fact that in many applications of games, the objects of interest that one wants to define and/or to realize algorithmically are really the winning strategies rather than the winning regions. In particular, this is the case when games are used to model reactive systems where the construction of winning strategies corresponds to the synthesis of controllers. Strategies can be viewed and presented in several different ways, and it is not always obvious what definability of strategies really means. However, for the games that we consider here, positional strategies suffice, and since we can identify a positional strategy with a set of edges in the game graph, definability questions for such games can be put very naturally, in terms of formulae strat $(x, y)$. Given the known results on definability of winning regions, and the results of finite model theory relating definability and (polynomial-time) complexity, by far the most natural logics for our purposes are fixed-points logics such as LFP and IFP. While the two problems of defining winning regions and winning strategies are closely related, they are not always equivalent. Indeed one can construct games where winning regions can be determined trivially, but winning strategies are not even computable (see Section VI). However, most algorithms that compute winning regions in games do so by revealing also winning strategies. Also from logical definitions of winning regions, one can often extract the underlying winning strategies. This is obvious in the case of a player with a safety objective who can win just by staying inside her winning region. For players with other objectives, such as reachability, natural LFP-definitions of winning regions associate with each position a rank, and winning strategies progress by reducing the rank. In such a case, winning strategies may be LFP-definable by rank comparison.

We shall study definability questions for positional winning strategies systematically in the following way. We shall first make precise what it means that a formula strat $(x, y)$ defines a winning strategy for a game. In a context of logical definability it is important to admit also nondeterministic strategies, rather than just deterministic ones, because in the presence of symmetries of the game graph, no logical formula can distinguish between moves that are mapped to each other by an automorphism of the game graph. We remark that the notion of nondeterministic strategies is well motivated also from a purely game-theoretic point of view, and by applications in controller synthesis and verification. In many cases it is important to design a winning strategy that is as permissive as possible in the sense that while it guarantees a win for the player, it still admits as much freedom as possible for the moves of the opponent and the plays that are consistent with the strategy, so as to eliminate, ideally, just the losing plays and keep as many as possible of the winning ones. We shall also briefly recall the background on the logics that we will employ here, in particular concerning least and inflationary fixed-point logic, LFP and IFP.

We shall then exhibit explicit definitions in fixed-point logic for winning strategies in games with relatively simple objectives, such as safety, reachability, eventual safety (Co- 
Büchi) and recurrent reachability (Büchi) before we establish our main result that winning strategies for any class of parity games with a bounded number of priorities are LFPdefinable. Our construction strongly depends on the Stage Comparison Theorem for LFP. We shall then consider the general question to what extent and under which conditions definable characterisations of winning regions permit logical translations into definable winning strategies, and vice versa. We show that this is, in general, a non-trivial question. Under natural conditions, the transfer from winning strategies to the corresponding winning regions is not so problematic, and we treat in particular the cases of parity games (with an unbounded number of priorities) and games with a fixed $\omega$ regular winning condition (depending on a bounded number of colours). As a consequence, it follows that winning strategies for parity games over infinite game graphs are, in general, not LFP-definable. Translations in the other direction are more delicate. We introduce a general concept of LFP-translations from winning regions to winning strategies on a class of game arenas. While there exist classes that do not admit such translations in general, we shall be able to prove that the class of finite game arenas does admit such translations. As a consequence it follows that there exist LFP-definable winning strategies on the class of all finite parity games (with an unbounded number of priorities) if, and only if, parity games can be solved in polynomial time, despite the fact that LFP is, in general, strictly weaker than polynomial time.

\section{PRELIMINARIES}

Games and strategies. We consider (two-player) games given by an $\omega$-coloured game arena that determines the possible moves and by a winning condition which is a set of $\omega$ coloured sequences that defines the winning plays for Player 0 . Whenever we study a class of games we assume that we have agreed on a fixed winning condition (such as reachability, Büchi, parity, and so on). Thus, when we represent games as mathematical structures we only specify the game arena but not the winning condition as part of the structure.

Game arenas are non-terminating graphs whose vertices are coloured by natural numbers. We represent them by two-sorted structures $G=\left(V \uplus \omega, V_{0}, V_{1}, E, \Omega,<\right)$ where $<$ is the usual order on the natural numbers $\omega$, where $\left(V, V_{0}, V_{1}, E\right)$ is a nonterminating graph and where $\Omega: V \rightarrow \omega$ is a function from the first sort (the vertices $V$, the vertex sort) to the second sort (the ordered set of natural numbers $\omega$, the colour sort). As usual, the vertex set $V=V_{0} \uplus V_{1}$ is partitioned into positions $V_{0}$ controlled by Player 0 and positions $V_{1}$ controlled by Player 1 . The edge relation $E \subseteq V \times V$ specifies the possible moves of the players. A play (starting at position $\left.v_{0} \in V\right)$ is an infinite $G$-path $\pi=v_{0} v_{1} \cdots \in V^{\omega}$ such that $\left(v_{i}, v_{i+1}\right) \in E$. With each such play we associate the induced colour sequence $\Omega(\pi)=\Omega\left(v_{0}\right) \Omega\left(v_{1}\right) \cdots \in \omega^{\omega}$. A winning condition (for Player 0 ) is a set $\mathcal{W} \subseteq \omega^{\omega}$. A game $\mathcal{G}$ is a tuple $\mathcal{G}=(G, \mathcal{W})$ consisting of a game arena $G$ and a winning condition $\mathcal{W}$. Player 0 wins a play $\pi \in V^{\omega}$ in $\mathcal{G}$ if $\Omega(\pi) \in \mathcal{W}$, otherwise $\pi$ is won by Player 1 .
A nondeterministic positional strategy for Player $\sigma$ in a game $\mathcal{G}=(G, \mathcal{W})$ is a set of edges $S \subseteq E \cap\left(V_{\sigma} \times V\right)$. The support of $S$ is $\operatorname{supp}(S):=\{v: v S \neq \emptyset\}$, and the closure of $S$ is $\hat{S}:=S \cup\left(E \cap V_{1-\sigma} \times V\right)$. Moreover, for $u \in V$ we let $\operatorname{Reach}(\hat{S}, u) \subseteq V$ denote the set of all nodes which are reachable from $u$ in $G$ via an $\hat{S}$-path. The strategy $S$ is a winning strategy (for Player $\sigma$ ) from $u \in V$ if $\left(\operatorname{Reach}(\hat{S}, u) \cap V_{\sigma}\right) \subseteq \operatorname{supp}(S)$ and if every $\hat{S}$-path $\pi$ starting in $u$ is a winning play for Player $\sigma$. The strategy $S$ is a winning strategy (for Player $\sigma$ ) on a set $U \subseteq V$ if $S$ is winning from every $u \in U$. We define the winning region of $S$ as the set $W(S)$ of nodes $u \in V$ such that $S$ is winning from $u$. We call a strategy $S$ for Player $\sigma$ complete if it is a winning strategy on the entire winning region of that player, i.e. if $W(S)=W_{\sigma}$.

Fixed-point logics. We are interested in the definability of winning regions and (positional) winning strategies in leastfixed point logic LFP over two-sorted game arenas $G=(V \uplus$ $\left.\omega, V_{0}, V_{1}, E, \Omega,<\right)$. We assume that the reader is familiar with fixed-point logics and their relationship with polynomial-time complexity (for background see e.g. [9, Chapters 2 and 3]) but we briefly recall the definitions of LFP and IFP, as well as the Stage Comparison Theorem for LFP.

Let $H: \mathcal{P}\left(V^{k}\right) \rightarrow \mathcal{P}\left(V^{k}\right)$ be an operator on $k$-ary relations over $V$. If $H$ is monotone (that is, $R \subseteq R^{\prime}$ implies $\left.H(R) \subseteq H\left(R^{\prime}\right)\right)$, then it has a least and a greatest fixedpoint. We define the stages $\left(H^{\beta}\right)_{\beta \in \mathrm{On}}$ of the least fixed-point induction by $H^{0}=\emptyset, H^{\beta+1}=H\left(H^{\beta}\right)$, and $H^{\lambda}=\bigcup_{\beta<\lambda} H^{\beta}$ for limit ordinals $\lambda$. There exists a smallest ordinal $\alpha$ such that $H^{\alpha}=H^{\alpha+1}=: H^{\infty}$ is the least fixed-point of $H$.

The logic LFP is built on top of first-order logic (FO) by adding least and greatest fixed points of definable relations: If $\varphi(R, \bar{x})$ is a formula with a new relation symbol $R$ of arity $k=|\bar{x}|$ and $\bar{x}$ is a tuple of first-order variables such that $R$ occurs only positively, then $[\operatorname{lfp} R \bar{x} \cdot \varphi(R, \bar{x})](\bar{y})$ is a formula of LFP. The semantics of such formulae are defined as the least fixed-points of the monotone operator $F_{\varphi}: \mathcal{P}\left(V^{k}\right) \rightarrow$ $\mathcal{P}\left(V^{k}\right), R \mapsto\{\bar{v}:(G, R) \models \varphi(R, \bar{v})\}$. We sometimes write $\varphi^{\alpha}$ for the $\alpha$-th stage of the least fixed-point induction of $F_{\varphi}$. We shall also use greatest fixed-point formulae of the form $[\operatorname{gfp} R \bar{x} \cdot \varphi(R, \bar{X})](\bar{y})$, whose semantics correspond to greatest fixed-points of the operators $F_{\varphi}$.

For operators $H$ which may or may not be monotone, we define the stages of the inflationary fixed-point induction by $\tilde{H}^{0}=\emptyset, \tilde{H}^{\beta+1}=\tilde{H}^{\beta} \cup H\left(\tilde{H}^{\beta}\right)$, and $\tilde{H}^{\lambda}=\bigcup_{\beta<\lambda} \tilde{H}^{\beta}$ for limit ordinals $\lambda$. Since the sequence of stages is increasing, inflationary fixed-point inductions always reach a fixed point $\tilde{H}^{\alpha}=\tilde{H}^{\alpha+1}=: \tilde{H}^{\infty}$. The logic IFP is defined similarly to LFP, but inflationary fixed points are used instead of least and greatest fixed points: If $\varphi(R, \bar{x})$ is a formula with a new relation symbol $R$ of arity $k=|\bar{x}|$ and $\bar{x}$ is a tuple of first-order variables (where $R$ may occur also negatively), then $[\operatorname{ifp} R \bar{x} \cdot \varphi(R, \bar{x})](\bar{y})$ is a formula, whose semantics is the inflationary fixed point of the operator $F_{\varphi}: R \mapsto\{\bar{v}:$ $(G, R) \models \varphi(R, \bar{v})\}$. 
For monotone operators, $\tilde{E}^{\infty}=E^{\infty}$. Hence, every formula of LFP can easily be translated into a formula of IFP. It is a deep result by Gurevich and Shelah [10] for the case of finite structures and by Kreutzer [15] for the general case, that also the converse holds, i.e. that LFP $=$ IFP. Although this fact justifies to use the two logics interchangeably, one should be aware that the translation of IFP-formulae into equivalent LFP-formulae is far from obvious and changes the structure and complexity of the formulae considerably. It is fair to say that LFP and IFP are really two different logics which happen to have the same expressive power. The main technical step in the proofs showing that $\mathrm{LFP}=\mathrm{IFP}$ is to express the stage comparison relations of IFP-inductions in LFP. In this paper we also make use of the LFP-definability of these relations, but for our purposes it suffices to consider these relations for the simpler case, due already to Moschovakis [16], of LFPinductions.

Let thus $G$ be an arena, let $\varphi(R, \bar{x})$ be a formula such that $R$ occurs only positively, and let $\bar{v}$ be a tuple from $V$ with the same arity as $R$ (and $\bar{x}$ ). The rank $|\bar{v}|_{\varphi}$ of $\bar{v}$ with respect to $\varphi$ is defined as the least ordinal $\alpha$ such that $\bar{v} \in \varphi^{\alpha}$. If there is no such ordinal, the rank of $\bar{v}$ is $\infty$. The stage comparison relations $\leq_{\varphi}$ and $\prec_{\varphi}$ of $\varphi$ are defined as

$$
\begin{gathered}
\bar{x} \leq_{\varphi} \bar{y} \text { if, and only if, } \bar{x}, \bar{y} \in \varphi^{\infty} \text { and }|\bar{x}|_{\varphi} \leq|\bar{y}|_{\varphi}, \\
\bar{x} \prec_{\varphi} \bar{y} \text { if, and only if, } \bar{x} \in \varphi^{\infty} \text { and }|\bar{x}|_{\varphi}<|\bar{y}|_{\varphi},
\end{gathered}
$$

where we allow $|\bar{y}|_{\varphi}$ to be $\infty$. For details on this and the following theorem, see [16], [10], [15].

Theorem 1 (Stage Comparison Theorem). For every LFPformula $\varphi(R, \bar{x})$ that is positive in $R$ there exist LFP-formulae $\left(\bar{x} \prec_{\varphi} \bar{y}\right)$ and $\left(\bar{x} \leq_{\varphi} \bar{y}\right)$ that define the stage comparison relations associated with $\varphi$. These formulae have the same alternation depth as $\varphi$ and their outermost fixed-point variable has twice the arity of $R$.

Definability of winning regions and winning strategies. Let $\mathcal{K}$ be a class of game arenas. We say that a winning condition $\mathcal{W} \subseteq \omega^{\omega}$ guarantees positional winning strategies for Player $\sigma$ on $\mathcal{K}$, if for every arena $G \in \mathcal{K}$, Player $\sigma$ has a complete positional winning strategy in the game $\mathcal{G}=(G, \mathcal{W})$ on his winning region. In this case we say that the pair $(\mathcal{K}, \mathcal{W})$ allows LFP-definable winning regions for Player $\sigma$ if there is an LFP-formula $\operatorname{win}_{\sigma}(x)$ which defines, in every game arena $G \in \mathcal{K}$, the winning region of Player $\sigma$ in the game $\mathcal{G}=(G, \mathcal{W})$. Analogously, $(\mathcal{K}, \mathcal{W})$ allows LFPdefinable (positional) winning strategies if there is an LFP-

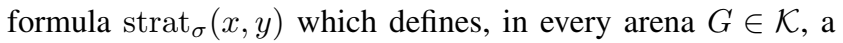
complete positional winning strategy for Player $\sigma$ in the game $\mathcal{G}=(G, \mathcal{W})$.

On two-sorted structures. Since game arenas are two-sorted structures, we consider (a variant of) two-sorted LFP over structures $G=\left(V \uplus \omega, V_{0}, V_{1}, E, \Omega,<\right)$. As usual for the two-sorted setting we have, for both, the vertex and the colour sort, a collection of typed first-order variables. We agree to use Latin letters $x, y, z, \ldots$ for variables ranging over the vertices and Greek letters $\nu, \mu, \ldots$ for variables ranging over the colours. Note that for every vertex variable $x, \Omega(x)$ is a term over the colour sort (indeed this is the only non-trivial kind of term which can be formed).

For second-order variables $R$ we allow mixed types. In order to restrict the expressive power of LFP over $(\omega,<)$ in a reasonable way and to ensure that the range of colour variables in quantifiers is finite, we require that quantification over the second sort is always bounded by a colour term, i.e. $Q \nu \leq t . \varphi$ where $Q \in\{\exists, \forall\}$ and where $t$ is a colour term in which $\nu$ does not occur free, i.e. $t=\Omega(x)$ or $t=\mu$ for $\mu \neq \nu$. Of course, the same restriction applies for fixed-point definitions. Still, our version of LFP is expressive enough to define important numerical properties of the colours like "the colour $\Omega(x)$ of vertex $x$ is even".

As we often deal with winning conditions over a finite set of colours (i.e., winning conditions of the form $\mathcal{W} \subseteq[d]^{\omega}$ for some $d \in \mathbb{N}$ ), we also view game arenas as one-sorted relational structures over the vocabulary $\tau_{d}=\left\{V_{0}, V_{1}, E, P_{0}, \ldots, P_{d-1}\right\}$, where $P_{0}, \ldots, P_{d-1}$ are unary predicates which encode the colours of the vertices. Obviously, the assumption that $d$ is a constant is crucial at this point. It is easy to see that in this situation both representations are equivalent (e.g., $P_{i} x$ translates to $\exists^{=i} \nu(\nu<\Omega(x))$ ). For better readability we will use the representation of game arenas as one-sorted structures in Section V knowing that our results can as well be formulated in the two-sorted setting.

\section{SAFETY AND REACHABILITY GAMES}

To discuss the problem of how to translate logical definitions of winning regions into definitions of complete winning strategies, we first focus on the simplest objectives for games on graphs, which are safety and reachability objectives. These are dual to each other: If we assume that players have strictly complementary goals, and the objective of one player is to reach a certain set $F$ of positions, then the opponent has a safety objective to ensure that the play stays inside the complement of $F$.

A reachability/safety game is thus given by a game graph $G$ and a subset $F \subseteq V$ that Player 0 wants to reach and Player 1 wants to avoid. The winning region for Player 0 is a least fixed point whereas the wining region for the safety player is a greatest fixed point. The winning regions can be defined by the formulae

$$
\begin{gathered}
\operatorname{win}_{0}(x):=\left[\operatorname{lfp} W x . F x \vee\left(V_{0} x \wedge \exists y(E x y \wedge W y)\right)\right. \\
\vee\left(V_{1} x \wedge \forall y(E x y \rightarrow W y)\right](x), \\
\operatorname{win}_{1}(x):=\left[\operatorname{gfp} W x . \neg F x \wedge\left(V_{0} x \rightarrow \forall y(E x y \rightarrow W y)\right)\right. \\
\left.\wedge\left(V_{1} x \rightarrow \exists y(E x y \wedge W y)\right)\right](x) .
\end{gathered}
$$

For the safety player, here Player 1 , it is trivial to extract a winning strategy from the winning region since all the player has to do is to remain inside his winning region. Thus, a complete winning strategy is defined by the formula

$$
\operatorname{strat}_{1}(x, y):=V_{1} x \wedge \operatorname{Exy} \wedge \operatorname{win}_{1}(y) .
$$


For the reachability player, here Player 0 , it does not suffice to remain inside the winning region; a winning strategy actually has to make progress towards the target set. However, the LFP-definition $\operatorname{win}_{0}(x)=[\operatorname{lfp} W x \cdot \varphi(W, x)](x)$ of the winning region $W_{0}$ gives us a (on infinite graphs possibly transfinite) stratification into stages $W_{0}=\bigcup_{\alpha \in \mathrm{On}} W_{0}^{\alpha}$ and associates with every position $v \in W_{0}$ the $\operatorname{rank} \operatorname{rk}(v):=|v|_{\varphi}$. We call a strategy $S$ for Player 0 strictly progressive if $\operatorname{rk}(v)<\operatorname{rk}(u)$ for all $(u, v) \in S$. It then follows from the definition of $\varphi$ that $\operatorname{rk}(v)<\operatorname{rk}(u)$ even holds for all $(u, v) \in \hat{S}$ with $\operatorname{rk}(u)<\infty$. Hence ranks are strictly decreasing on all plays that start in $W(S)$ and are consistent with $S$. As a consequence, the union of two strictly progressive winning strategies is again strictly progressive, which implies that there exists a unique maximal strictly progressive winning strategy for Player 0, which we call the optimal winning strategy. It can be described as the set of edges from $V_{0} \times V$ that strictly decrease the rank.

We infer that not only the winning regions in reachability games are definable in fixed-point logic, but also the optimal winning strategies. In fact we obtain a simultaneous IFPdefinition of the winning regions and the optimal winning strategy $S^{*}$. Indeed the pair $\left(W_{0}, S^{*}\right)$ is the simultaneous fixed-point of the IFP-formula

$$
\begin{aligned}
\operatorname{ifp}(W, S) . W x \leftarrow & F x \vee\left(V_{0} x \wedge \exists y(E x y \wedge W y)\right) \\
& \vee\left(V_{1} x \wedge \forall y(E x y \rightarrow W y)\right) \\
S x y \leftarrow & E x y \wedge V_{0} x \wedge \neg W x \wedge W y .
\end{aligned}
$$

For an alternative possibility for defining optimal winning strategies, we directly use the stage comparison relation which, by the Stage Comparison Theorem, is definable by an LFP-formula $\prec_{\varphi}$. It follows that optimal winning strategies in reachability games are defined by the LFP-formula $\operatorname{strat}_{0}(x, y):=V_{0} x \wedge E x y \wedge y \prec_{\varphi} x$. The following theorem summarizes the observations of this section.

Theorem 2. For safety games, winning regions and complete winning strategies are definable by LFP-formulae with just one monadic greatest fixed point operator, applied to a firstorder formula. For reachability games, winning regions and complete winning strategies are definable by LFP-formulae with binary least fixed-points operators, applied to first-order formulae.

\section{BÜCHI GAMES}

In a recurrent reachability or Büchi game, Player 0 tries to enforce an infinite number of visits to a target set $F$. The opponent has an eventual safety, or Co-Büchi, objective, trying to ensure that from some point onwards the target set $F$ is avoided. We can regard Büchi games as parity games over the set of priorities $\{0,1\}$. We shall prove that, on the basis of LFP-formulae $\operatorname{win}_{0}(x)$ and $\operatorname{win}_{1}(x)$ that define the winning regions in Büchi games, we can construct formulae $\operatorname{strat}_{0}(x, y)$ and $\operatorname{strat}_{1}(x, y)$ defining complete positional winning strategies for the two players. The ideas and techniques that we use will generalise to parity games with a bounded number of priorities. Büchi games can be solved by nested attractor computations. The winning region of Player 0 in a Büchi game with target set $F$ is defined by the formula

$$
\begin{aligned}
\operatorname{win}_{0}(x):= & {[\operatorname{gfp} Y y \cdot[\operatorname{lfp} Z z \cdot \varphi(Y, Z, z)](y)](x) \text { where } } \\
\varphi(Y, Z, z):= & \left(F z \wedge \left(\left(V_{0} z \wedge \exists u(E z u \wedge Y u)\right)\right.\right. \\
& \left.\left.\vee\left(V_{1} z \wedge \forall u(E z u \rightarrow Y u)\right)\right)\right) \\
& \vee\left(\neg F z \wedge \left(\left(V_{0} z \wedge \exists u(E z u \wedge Z u)\right)\right.\right. \\
& \left.\left.\vee\left(V_{1} z \wedge \forall u(E z u \rightarrow Z u)\right)\right)\right) .
\end{aligned}
$$

The winning strategy underlying this formula has two components. At nodes in $F$, Player 0 just has to ensure to stay in her winning region. Thus, she plays with a safety strategy, and we need nothing more than the formula win w $_{0}$ to define her winning strategy at positions in $F$. At nodes outside $F$, Player 0 needs to make progress towards a node in $F$ that is in her winning region. She is able to do that by employing an attractor strategy that is based on the stratification $\bigcup_{\alpha \in \text { On }} W_{0}^{\alpha}$ of her winning region $W_{0}$. For $v \in W_{0}$, we have $v \in W_{0}^{0}$ if, and only if, $v$ is in $F$. This attractor strategy underlies the last least fixed-point induction in the evaluation of the formula $\operatorname{win}_{0}$. The strategy is defined, as for reachability games, by a stage comparison relation. We need to define a formula that induces the correct least fixed-point induction and its associated stage comparison relation. Given the inner subformula $\varphi(Y, Z, z)$ inside the the formula $\operatorname{win}_{0}(x)$, we put $\varphi^{*}(Z, z):=\varphi(Y, Z, z)\left[Y u / \operatorname{win}_{0}(u)\right]$ where we replace in $\varphi$ every occurrence of the gfp-variable $Y$ by the formula defining that greatest fixed point. Thus $\varphi^{*}(Z, z)$ defines the update operator for the lfp-induction at points outside $F$, for the case that $Y$ is set to the winning region.

By the Stage Comparison Theorem, the associated stage comparison relation is definable by an LFP-formula $x \prec_{\varphi^{*}} y$. Thus a complete winning strategy for Player 0 in a Büchi game is defined by the formula $\operatorname{strat}_{0}(x, y):=$

$$
V_{0} x \wedge \operatorname{Exy} \wedge\left(\left(F x \wedge \operatorname{win}_{0}(y)\right) \vee\left(\neg F x \wedge y \prec_{\varphi^{*}} x\right)\right) .
$$

For Player 1, who plays with the Co-Büchi objective to hit $F$ only finitely often, the winning region is defined by a dual formula

$$
\begin{aligned}
\operatorname{win}_{1}(x):= & {[\operatorname{lfp} Y y \cdot[\operatorname{gfp} Z z \cdot \vartheta(Y, Z, z)](y)](x) \text { where } } \\
\vartheta(Y, Z, z):= & \left(F z \wedge \left(\left(V_{1} z \wedge \exists u(E z u \wedge Y u)\right)\right.\right. \\
& \left.\left.\vee\left(V_{0} z \wedge \forall u(E z u \rightarrow Y u)\right)\right)\right) \\
& \vee\left(\neg F z \wedge \left(\left(V_{1} z \wedge \exists u(E z u \wedge Z u)\right)\right.\right. \\
& \left.\left.\vee\left(V_{0} z \wedge \forall u(E z u \rightarrow Z u)\right)\right)\right) .
\end{aligned}
$$

Again, we define the positional winning strategy that underlies $\operatorname{win}_{1}(x)$ by stage comparison relations. The winning region of Player 1 has a stratification $W_{1}=\bigcup_{\alpha \in \text { On }} W_{1}^{\alpha}$ which assigns to every position $v$ a $\operatorname{rank} \operatorname{rk}(v):=\min \left\{\alpha: v \in W_{1}^{\alpha}\right\}$ if $v \in W_{1}$, and $r k(v)=\infty$ otherwise. On each stage $W_{1}^{\alpha}$, Player 1 is able to trap the play inside the set $\bigcup_{\beta \leq \alpha} W_{1}^{\beta}$, and at nodes $v \in F$ of rank $\alpha$ he can force the play to a successor position $u$ of strictly smaller rank. The set $W_{1}^{0}$ contains only 
positions outside $F$. Thus, on her winning region $W_{1}$, Player 1 can ensure that, from some point onwards, only positions outside $F$ are visited. The formula defining the operator for the lfp-induction is $\varphi(Y, y):=[\operatorname{gfp} Z z . \vartheta(Y, Z, z)](y)$ and the stratification $\left(W_{1}^{\alpha}\right)_{\alpha \in \text { On }}$ coincides with the stages of the LFP-induction of $\varphi(Y, y)$. The associated winning strategy can now be expressed in terms of the LFP-formulae $x \prec_{\varphi} y$ and $x \leq_{\varphi} y$ for the stage comparison relations with respect to $\varphi$, by the formula $\operatorname{strat}_{1}(x, y):=$

$$
V_{1} x \wedge \operatorname{Exy} \wedge\left(\left(F x \wedge y \prec_{\varphi} x\right) \vee\left(\neg F x \wedge y \leq_{\varphi} x\right)\right) .
$$

Theorem 3. On Büchi games complete winning strategies for both players are definable by LFP-formulae of alternation depth two with binary fixed-point variables.

\section{Parity Games With a Bounded Number of PRIORITIES}

The formulae defining winning regions for Büchi and CoBüchi games can be generalised to LFP-definitions of winning regions for parity games with priorities in $\{0, \ldots, d-1\}$, for any fixed $d \in \mathbb{N}$. We regard such games as relational structures over the vocabulary $\tau_{d}:=\left\{V_{0}, V_{1}, E, P_{0}, \ldots, P_{d-1}\right\}$, defined as in Section II, and denote this class by $\mathcal{P} \mathcal{G}_{d}$.

Theorem 4. The winning region of Player 0 in parity games in $\mathcal{P} \mathcal{G}_{d}$ is defined by $\operatorname{win}_{0}^{d}\left(x_{0}\right):=$

$\left[\operatorname{gfp} X_{0} x_{1} \cdot\left[\operatorname{lfp} X_{1} x_{2} \cdots\left[\mathbf{f p} X_{d-1} x_{d} \cdot \varphi_{d}\right]\left(x_{d-1}\right) \cdots\right]\left(x_{1}\right)\right]\left(x_{0}\right)$

with

$$
\begin{array}{r}
\varphi_{d}\left(\bar{X}, x_{d}\right):=\bigvee_{k=0}^{d-1}\left(\left(V_{0} x_{d} \wedge P_{k} x_{d} \wedge \exists y\left(E x_{d} y \wedge X_{k} y\right)\right) \vee\right. \\
\left.\left(V_{1} x_{d} \wedge P_{k} x_{d} \wedge \forall y\left(E x_{d} y \rightarrow X_{k} y\right)\right)\right)
\end{array}
$$

The fixed-point operators alternate between greatest and least fixed-points; hence, the innermost fixed-point is a greatest fixed-point if $d$ is odd, and a least fixed-point if $d$ is even.

This construction is due to Walukiewicz [21]. For a different proof of correctness, based on model checking games, see [9, Chapter 3.3.6]. Our goal is to show that on every class $\mathcal{P} \mathcal{G}_{d}$, not just the winning regions, but also complete winning strategies are LFP-definable.

Theorem 5. For every $d \in \mathbb{N}$, there exists an LFPformula $\operatorname{strat}_{0}^{d}(x, y)$ that defines a complete winning strategy for Player 0 for parity games in $\mathcal{P} \mathcal{G}_{d}$.

Our approach for proving this generalises the construction for reachability, safety, and Büchi games. We take the formula for defining winning regions and extract from it a formula that defines a winning strategy, based on stage comparison arguments. However, the formula defining the winning regions has alternation depth $d$, which makes the construction considerably more involved.

We have to take apart, and modify the formula $\operatorname{win}_{0}^{d}$, so as to obtain for each priority $k<d$ an appropriate inductive operator (with only one free fixed-point variable) on which we can apply the Stage Comparison Theorem.

Starting with the first-order part $\varphi_{d}\left(\bar{X}, x_{d}\right)$ of the formula $\operatorname{win}_{0}^{d}\left(x_{0}\right)$, we inductively put $\varphi_{k}\left(X_{0}, \ldots, X_{k-1}, x_{k}\right):=$ $\left[\mathbf{f p} X_{k} x_{k+1} \cdot \varphi_{k+1}\left(X_{0}, \ldots, X_{k}, x_{k+1}\right)\right]\left(x_{k}\right)$ where fp $=\mathbf{g f p}$ in case $k$ is even and $\mathbf{f p}=\mathbf{l f p}$ for odd $k$. Notice that $\varphi_{0}\left(x_{0}\right)$ coincides with $\operatorname{win}_{0}^{d}\left(x_{0}\right)$ and thus defines the winning region of Player 0.

Based on the formula $\varphi_{k}\left(X_{0}, \ldots, X_{k-1}, x_{k}\right)$, we construct formulae $\psi_{k}$ to define Player 0's next moves from positions with priority $k$. To do so, we have to eliminate the free secondorder variables in $\varphi_{k}$ in the right manner and find the correct induction for every position in the game, based on the stage comparison relation. The formulae $\psi_{0}$ and $\psi_{1}$ contain one free first-order variable, the formulae $\psi_{2}, \ldots, \psi_{d-1}$ contain a second free first-order variable $z$ that is a parameter. The formulae $\psi_{k}$, for odd $k$, begin with a least fixed-point operator. Therefore, there is a stage comparison relation associated with its induction. We write $\prec_{1}$ and $\leq_{1}$ for the stage comparison relations with respect to $\psi_{1}$, and $\prec_{k}^{z}$ and $\leq_{k}^{z}$ with respect to $\psi_{k}$ for odd $k \in\{3, \ldots, d-1\}$, where is $z$ is the parameter of $\psi_{k}$. These stage comparison relations are definable in LFP by the Stage Comparison Theorem.

- $\psi_{0}\left(x_{0}\right):=\varphi_{0}\left(x_{0}\right)$,

- $\psi_{1}\left(x_{1}\right):=\varphi_{1}\left(x_{1}, X_{0} u / \varphi_{0}(u)\right)$,

- $\psi_{2}\left(z, x_{2}\right):=\varphi_{2}\left(x_{2}, X_{0} u / \varphi_{0}(u), X_{1} u / u \prec_{1} z\right)$, and

- for $2<k<d$ : $\psi_{k}\left(z, x_{k}\right)$ is defined as $\varphi_{k}$ with every occurrence of $X_{j} u$, for $0 \leq j<k$ even, substituted by $\psi_{j}(z, u)$, and every occurrence of $X_{j} u$, for $0 \leq j<k$ odd, substituted by $u \prec_{j}^{z} z$.

To distinguish between $x_{k}$ and the parameter $z$ we write $\psi_{k, z}\left(x_{k}\right)$ instead of $\psi_{k}\left(z, x_{k}\right)$. We are ready to define the LFP-formula that expresses Player 0's winning strategy $\operatorname{strat}_{0}^{d}(x, y):=$

$$
\begin{gathered}
V_{0} x \wedge \operatorname{Exy} \wedge\left(\left(P_{0} x \wedge \psi_{0}(y)\right) \vee \bigvee_{\substack{1<k<d, k \text { even }}}\left(P_{k} x \wedge \psi_{k, x}(y)\right) \vee\right. \\
\left.\left(P_{1} x \wedge y \prec_{1} x\right) \vee \bigvee_{\substack{1<k<d, k \text { odd }}}\left(P_{k} x \wedge y \prec_{k}^{x} x\right)\right) .
\end{gathered}
$$

It remains to show that it indeed describes a complete winning strategy. Notice that $\psi_{k, z}\left(x_{k}\right)$ has the form $\left[\mathbf{f p} X_{k} x_{k+1} \cdot \vartheta_{k, z}\left(X_{k}, x_{k+1}\right)\right]\left(x_{k}\right)$. The following observation follows directly from the definition of $\psi_{k+1, z}$.

Lemma 6. If $\psi_{k, z}\left(x_{k}\right)=\left[\mathbf{f p} X_{k} x_{k+1} \cdot \vartheta_{k, z}\left(X_{k}, x_{k+1}\right)\right]\left(x_{k}\right)$, then for all positions $v, w$ in a game $\mathcal{G} \in \mathcal{P} \mathcal{G}_{d}$, we have

- for $k$ odd, $\mathcal{G}=\vartheta_{k, v}\left(\left\{u \in V: u \prec_{k}^{v} v\right\}, w\right) \Leftrightarrow \mathcal{G} \models$ $\psi_{k+1, v}(w)$, and

- for $k$ even, $\mathcal{G} \models \vartheta_{k, v}\left(\left\{u \in V: \mathcal{G} \models \psi_{k, v}(u)\right\}, w\right) \Leftrightarrow$ $\mathcal{G} \models \psi_{k+1, v}(w)$.

We next define the rank of a position $v \in W_{0}$. The difference to the ranks used in Büchi games is that the nested fixed-points in $\operatorname{win}_{0}^{d}$ induce a multi-dimensional rank, that is 
described by an $m$-tuple of ordinals, where $m:=\left\lceil\frac{d-1}{2}\right\rceil$ is the number of odd priorities in $\mathcal{G}$.

Definition 7. For a position $v \in W_{0}$ in a parity game $\mathcal{G} \in$ $\mathcal{P} \mathcal{G}_{d}$, let $\operatorname{rk}(v):=\left(\alpha_{1} \ldots \alpha_{m}\right)$ where $\alpha_{i}:=|v|_{\vartheta_{2 i-1, v}}$, i.e., the stage of $v$ in the induction of the formula $\vartheta_{k, v}\left(X_{k}, x_{k+1}\right)$ for $k=2 i-1$. For every $i \leq m$, we write $<_{i}$ for the lexicographical order on the components 1 to $i$ of such $m$ dimensional ranks.

For the rank to be well-defined, we need to show that, for $v \in W_{0}$, each component of the rank is indeed an ordinal.

Lemma 8. For all $k \in\{0, \ldots, d-1\}$ we have $v \in W_{0}$ if, and only if, $\mathcal{G} \models \psi_{k, v}(v)$.

Proof. Let $k \in\{0, \ldots, d-2\}$. We show that $\mathcal{G} \models \psi_{k, v}(v)$ if, and only if, $\mathcal{G}=\psi_{k+1, v}(v)$. Then, the claim follows directly, since we have $\mathcal{G}=\psi_{0}(v)$ if, and only if, $v \in W_{0}$.

If $k$ is even, we have $\psi_{k, z}=$ $\left[\operatorname{gfp} X_{k} x_{k+1} \cdot \vartheta_{k, z}\left(X_{k}, x_{k+1}\right)\right]\left(x_{k}\right)$. Let $A \subseteq V$ be the greatest fixed-point of $\vartheta_{k, v}$. In particular, we have

$$
A=\left\{u \in V: \mathcal{G} \models \vartheta_{k, v}(A, u)\right\}=\left\{u \in V: \mathcal{G} \models \psi_{k, v}(u)\right\} .
$$

It follows by Lemma 6 that

$\mathcal{G} \models \psi_{k, v}(v) \Leftrightarrow v \in A \Leftrightarrow \mathcal{G} \models \vartheta_{k, v}(A, v) \Leftrightarrow \mathcal{G} \models \psi_{k+1, v}(v)$.

If $k$ is odd, we have $\psi_{k, z}=$ $\left[\operatorname{lfp} X_{k} x_{k+1} \cdot \vartheta_{k, z}\left(X_{k}, x_{k+1}\right)\right]\left(x_{k}\right)$. Let $\left(X^{\beta}\right)_{\beta \in \text { On }} \quad$ be the stages of the least fixed-point induction of $\vartheta_{k, v}$. We have

$$
X^{\alpha}=\left\{u \in V: \mathcal{G} \models \vartheta_{k, v}\left(X^{\alpha-1}, u\right)\right\}
$$

for successor ordinals $\alpha . \mathcal{G} \equiv \psi_{k, v}(v)$ implies the existence of a successor ordinal $\alpha$ with $v \in X^{\alpha}$ and $v \notin X^{\alpha-1}$. Furthermore, we have $X^{\alpha}=\left\{u \in V: u \leq_{k}^{v} v\right\}$, and $X^{\alpha-1}=\left\{u \in V: u \prec_{k}^{v} v\right\}$. It follows by Lemma 6 that

$$
\begin{aligned}
v \in X^{\alpha} & \Leftrightarrow \mathcal{G} \models \vartheta_{k, v}\left(\left\{u \in V: u \prec_{k}^{v} v\right\}, v\right) \\
& \Leftrightarrow \mathcal{G} \models \psi_{k+1, v}(v) .
\end{aligned}
$$

Assume $\mathcal{G} \not \models \psi_{k, v}(v)$. Then, we have $v \notin X^{\infty}$ and $X^{\infty}=$ $\left\{u \in V: u \prec_{k}^{v} v\right\}$. It follows by Lemma 6 that

$$
\begin{aligned}
v \notin X^{\infty} & \Leftrightarrow \mathcal{G} \not \vartheta_{k, v}\left(\left\{u \in V: u \prec_{k}^{v} v\right\}, v\right) \\
& \Leftrightarrow \mathcal{G} \not=\psi_{k+1, v}(v) .
\end{aligned}
$$

Altogether, we have shown $\mathcal{G} \models \psi_{k, v}(v)$ if, and only if, $\mathcal{G} \models \psi_{k+1, v}(v)$, for all $0 \leq k<d-1$.

Thus, the rank function is indeed well-defined. In the case of reachability games, we argued that our winning strategy $S$ was defined correctly, because, in a play that is consistent with $S$, the ranks are strictly decreasing until the set $F$ is visited. The following lemma is an analogue to that observation.

Lemma 9. Let $S$ be the strategy defined by the formula $\operatorname{strat}_{0}^{d}(x, y)$, and let $v \in W_{0}$ be a position with priority $k$. Then, the following three statements hold.

1) If $v \in V_{0}$, then $v S \neq \emptyset$.

2) We have $w \in W_{0}$ for all $w \in v \hat{S}$.
3) If $k$ is odd, $\operatorname{rk}(w)<_{\frac{k+1}{2}} \operatorname{rk}(v)$ for all $w \in v \hat{S}$, and if $k$ is even, $\operatorname{rk}(w) \leq_{\frac{k}{2}} \operatorname{rk}(v)$ for all $w \in v \hat{S}$.

Proof. We prove the first claim. Let $v \in V_{0}, k$ be odd, and $\left(X^{\beta}\right)_{\beta \in \text { On }}$ the sequence defined by the induction of $\vartheta_{k, v}$ on $\mathcal{G}$. We have $\mathcal{G}=\psi_{k, v}(v)$ by Lemma 8 . Hence, there is a successor ordinal $\alpha>1$ with with $v \in X^{\alpha}$ and $v \notin X^{\alpha-1}$. Since $X^{\alpha}=\left\{u \in V: \mathcal{G} \models \vartheta_{k, v}\left(X^{\alpha-1}, u\right)\right\}$ and by the definition of $\vartheta_{k, v}$, there is a position $w \in X^{\alpha-1}$ with $(v, w) \in E$, in particular $w \prec_{k}^{v} v$. We conclude $v S \neq \emptyset$.

Now, let $k$ be even. Again we have $\mathcal{G}=\psi_{k, v}(v)$, which means that $v$ is an element of the greatest fixed-point $A \subseteq V$ of $\vartheta_{k, v}$. It follows from the definition of $\vartheta_{k, v}$ that there is a position $w \in A$ with $(v, w) \in E$. Altogether, we conclude $v S \neq \emptyset$ for all $v \in V_{0}$.

We show the second and third claim. The problem is that $\operatorname{rk}(w)$ and $\operatorname{rk}(v)$ are defined by different formulae, namely $\psi_{1}, \psi_{3, w}, \ldots, \psi_{m, w}$ and $\psi_{1}, \psi_{3, v}, \ldots, \psi_{m, v}$ respectively. We have to show that both ranks can be compared nonetheless. Let $v$ be an arbitrary position in $W_{0}$ with priority $k$. For odd $1 \leq i \leq k$, we define the set of positions $U_{i}:=$ $\left\{u \in V: u \leq_{i}^{v} v\right\}$, and for even $1 \leq i \leq k$ we define $U_{i}:=\left\{u \in V: \mathcal{G} \models \psi_{i, v}(u)\right\}$. For $i<k$ odd, we have by Lemma 6 that

$$
\begin{aligned}
U_{i} & =\left\{u \in V: u \leq_{i}^{v} v\right\}= \\
& =\left\{u \in V: \mathcal{G} \models \vartheta_{i, v}\left(\left\{w \in V: w \prec_{i}^{v} v\right\}, u\right)\right\} \\
& =\left\{u \in V: \mathcal{G} \models \psi_{i+1, v}(u)\right\}=U_{i+1} .
\end{aligned}
$$

Let $i<k$ be even. We claim $U_{i+1} \subseteq U_{i}$ : Let $u \in U_{i+1}$. By definition we have $u \leq_{i+1}^{v} v$, which implies, by Lemma 6 , that

$$
\begin{aligned}
\mathcal{G} \models \psi_{i+1, v}(u) & \Leftrightarrow \mathcal{G}=\vartheta_{i, v}\left(\left\{w \in V: \mathcal{G}=\psi_{i, v}(w)\right\}, u\right) \\
& \Leftrightarrow \mathcal{G}=\psi_{i, v}(u) .
\end{aligned}
$$

Thus, we have $u \in U_{i}$.

If $k$ is odd, we conclude for all $1 \leq i<k$,

$$
\left\{u \in V: u \prec_{k}^{v} v\right\} \subsetneq\left\{u \in V: u \leq_{k}^{v} v\right\} \subseteq U_{i},
$$

and if $k$ is even, we conclude for all $1 \leq i<k$,

$$
\left\{u \in V: \mathcal{G} \models \psi_{k, v}(u)\right\} \subseteq U_{i} .
$$

Let $k$ be odd. If $v$ is controlled by Player 1 , we have $w \prec_{k}^{v} v$ for all $w \in v E=v \hat{S}$ by definition of $\psi_{k, v}$. If $v$ is controlled by Player 0 , we have $w \prec_{k}^{v} v$ for all $w \in v S=v \hat{S}$ by the definitions of $\psi_{k, v}$ and strat ${ }_{0}^{d}$. Let $w \in V$ with $w \prec_{k}^{v} v$, for an arbitrary $v \in W_{0}$. We need to show $\operatorname{rk}(w)<_{\frac{k+1}{2}} \operatorname{rk}(v)$. By the argument above, we have $w \in U_{i}$ for all $i \in\{1, \ldots, k-1\}$. In particular, this implies $w \in W_{0}$ for all $w \in v \hat{S}$, since we have $U_{1} \subseteq W_{0}$. If $w \prec_{1} v$, we are done. Otherwise, we have $U_{1}=\left\{u \in V: u \leq_{1} w\right\}$, which means $\psi_{2, v}$ and $\psi_{2, w}$ are equivalent on the game $\mathcal{G}$, and so are $\psi_{3, v}$ and $\psi_{3, w}$. Therefore, we can repeat the argument with $\psi_{3, v}$. Finally, we obtain

$$
\begin{aligned}
\left\{u \in V: u \leq_{k}^{w} w\right\} & =\left\{u \in V: u \leq_{k}^{v} w\right\} \\
& \subsetneq\left\{u \in V: u \leq_{k}^{v} v\right\}=U_{k},
\end{aligned}
$$


which means $r k(w)<_{\frac{k+1}{2}} r k(v)$ for all $w \in v \hat{S}$.

Let $k$ be even. If $v$ is controlled by Player 1 , we have $\mathcal{G}=\psi_{k, v}(w)$ for all $w \in v E=v \hat{S}$ by definition of $\psi_{k, v}$. If $v$ is controlled by Player 0 , we have $\mathcal{G} \models \psi_{k, v}(w)$ for all $w \in v S=v \hat{S}$ by definitions of $\psi_{k, v}$ and strat ${ }_{0}^{d}$. Thus, we have $w \in U_{i}$ for all $i \in\{1, \ldots, k-1\}$ by the argument above. In particular, this implies $w \in W_{0}$ for all $w \in v \hat{S}$. The rest of the argument works almost analogously to the case for odd $k$. With the exception, that at the end we might only conclude

$$
\left\{u \in V: \mathcal{G} \models \psi_{k, w}(u)\right\}=\left\{u \in V: \mathcal{G} \models \psi_{k, v}(u)\right\}=U_{k} .
$$

So we have $\operatorname{rk}(w) \leq_{\frac{k}{2}} r k(v)$ for all $w \in v \hat{S}$. This completes the proof of the lemma.

We are now ready to prove the correctness of the formula strat ${ }_{0}^{d}(, x, y)$, and thus Theorem 5. This is an immediate consequence of the following lemma.

Lemma 10. On a game $\mathcal{G} \in \mathcal{P} \mathcal{G}_{d}$, let $S$ be the strategy defined by the formula strat ${ }_{0}^{d}(x, y)$ and let $\pi=v_{0} v_{1} v_{2} \ldots$ be an $\hat{S}$ path that starts in the winning region of Player 0 , i.e. $v_{0} \in W_{0}$. Then $\pi$ is a winning play for Player 0.

Proof. By Lemma 9, $\pi$ remains in the winning region of Player 0 . We consider the sequence of ranks $\left(\operatorname{rk}\left(v_{i}\right)\right)_{i \in \mathbb{N}}$ induced by the play $\pi$. By Lemma 9 , we know that we have, for all $i \in \mathbb{N}$,

- $\operatorname{rk}\left(v_{i+1}\right)<_{\frac{k+1}{2}} \operatorname{rk}\left(v_{i}\right)$ for $k=\Omega\left(v_{i}\right)$ odd, and

- $\operatorname{rk}\left(v_{i+1}\right) \leq_{\frac{k}{2}}^{2} \operatorname{rk}\left(v_{i}\right)$ for $k=\Omega\left(v_{i}\right)$ even.

We take a closer look at the highest component $\ell$ in the sequence of $m$-tuples such that from some point onwards the first $\ell$ components of the ranks do not change anymore, more formally, the highest $1 \leq \ell \leq m$ such that there is a $j \in \mathbb{N}$ such that we have, for all $i \geq j$ and all $n \leq \ell$, $\operatorname{rk}\left(v_{i}\right)(n)=\operatorname{rk}\left(v_{i+1}\right)(n)$.

If no such $\ell$ exists, then the first component of the ranks changes infinitely often. But there is no infinite strictly decreasing chain of ordinals. Furthermore, the first position of a rank can only be increased if the play reaches a position with priority 0 . Thus, for the first position to change infinitely often a position with priority 0 has to be visited infinitely often. Hence, Player 0 wins the play.

Assume such an $\ell$ exists, and let $j$ be the corresponding position in the play, such that $\operatorname{rk}\left(v_{i}\right)(n)=\operatorname{rk}\left(v_{i+1}\right)(n)$ for all $i \geq j$ and all $n \leq \ell$, i.e. that from position $j$ onwards the first $\ell$ components in the tuples do not change. Thus, after position $v_{j}$ no position with an odd priority lower than $2 \ell$ is visited anymore. If we have $\ell=m$, no odd priority is seen from positions $j$ onwards, which means a win for Player 0 . In the case of $\ell<m$, the component $\ell+1$ changes infinitely often. Thus, it must also be increased an infinite number of times. But without changing a component greater than $\ell$, this can only be established by visiting a position with an even priority lower than $2 \ell+1$, which again means that Player 0 wins. Hence, Player 0 wins the play $\pi$ in every case.

\section{TRANSLATIONS BETWEEN WINNING REGIONS AND WINNING STRATEGIES}

Obviously the two fundamental concepts for the analysis of a game, winning regions and winning strategies, are intimately connected. Many algorithmic and logical approaches to games can be used to produce, with minor modifications, both winning regions and winning strategies. However this is not always the case and the passage from winning strategies to winning regions, and even more so in the other direction, is far from being trivial.

Indeed, for both directions there are examples of games where one concept is trivial, while the other is not computable. Such examples can be produced in many variations and need not be complex; there are automatic game graphs obtained from configuration graphs of Turing machines [6] such that the winning condition is trivial, while finding strategies corresponds to solving the halting problem, and, by simple modifications, such that all strategies of one player are winning, despite the winning region forming an undecidable set of vertices.

Theorem 11. There exist games $\mathcal{G}, \mathcal{G}^{\prime}$ over infinite automatic game graphs and winning conditions that are expressed in LTL or, equivalently, in first-order logic, such that for $\mathcal{G}$, the winning regions are computable but winning strategies are not, and for $\mathcal{G}^{\prime}$, complete winning strategies for both players are computable but winning regions are not.

Proof. Let $M$ be a (deterministic) Turing machine operating on natural numbers, with the following properties.

- The problem whether, for given $n \in \omega, M$ halts on input $n$, is undecidable.

- From $C_{0}(n)$, the initial configuration on input $n, M$ either reaches an accepting configuration, or the computation of $M$ is infinite without reaching a final configuration.

It is well-known that the configuration graph of any Turing machine is automatic. Let $H$ be the configuration graph of $M$ where the accepting configurations get an additional self-loop and are colored red, an let $H^{\prime}$ be a copy of $H$ with a switched coloring, i.e. precisely the non-accepting configurations are colored red.

Let now $\mathcal{G}$ be the game where Player 1 first picks any blue node $n \in \omega$, from which Player 0 has the choice to move to the input configuration $C_{0}(n)$ in either $H$ or in its copy $H^{\prime}$. We can assume that all nodes in $H \cup H^{\prime}$ belong to Player 1.

Now consider the LTL winning condition blue $\rightarrow$ FGred for Player 0, saying that if the play ever goes through a blue node then it will later reach a red node and never leave the red nodes after that.

With this winning condition, Player 0 has a winning strategy in $G$ from any position, since for every $n \in \omega$ it is the case that in either $H$ or $H^{\prime}$, the unique infinite path from $C_{0}(n)$ satisfies $F G$ red. Hence the winning region of Player 0 is the entire game graph. However, to find the winning strategy from node $n$ one has to decide whether $M$ halts on $n$, which is 
undecidable. Hence there is no computable winning strategy for Player 0 .

The winning condition can be formulated in several other ways, for instance blue $\rightarrow G F$ red. If one chooses a different colouring in $H^{\prime}$ one can also bring it to a form blue $\rightarrow(F$ red $\vee$ $G$ yellow), and express it by a first-order formula that is even in $\Delta_{2}$.

The construction of $\mathcal{G}^{\prime}$ is even simpler: just take the disjoint collection of all computation paths of $M$ from $C_{0}(n)$ for all natural numbers $n$, assuming that all moves (which are trivial anyway) are made by Player 1 . A play is won by Player 0 if it terminates, i.e., reaches a halting configuration. There are no choices so (winning) strategies are trivial, but the winning regions are undecidable.

Hence, for definability issues as studied in this paper, the question arises under what circumstances a logical definition of the winning regions in a class of games also yields a definition of complete winning strategies, and vice versa.

For the translation from winning strategies to winning regions, we give answers for two slightly different instances of the problem: One answers the question for certain classes of games, while the other provides a translation from winning strategies to winning regions for games with a single fixed $\omega$ regular winning condition. (Note that the parity condition is only $\omega$-regular for a bounded number of priorities.) The main ingredient in the proofs of the theorem are lemmas which state that the set of positions from where all paths consistent with a strategy satisfy the winning condition is definable in LFP.

Theorem 12. (a) Let $\mathcal{K} \subseteq \mathcal{P G}$ be a subclass of the class $\mathcal{P G}$ of all parity games (with an unbounded number of priorities) such that complete winning strategies are uniformly LFP-definable on $\mathcal{K}$. Then the winning regions are LFP-definable on $\mathcal{K}$.

(b) Let $\mathcal{W} \subseteq \Sigma^{\omega}$ be an $\omega$-regular language over a finite set $\Sigma$ of symbols. Let $\mathcal{A}$ be a class of arenas $G$ such that complete winning strategies for the class $\mathcal{K}=\{(G, \mathcal{W})$ : $G \in \mathcal{A}\}$ are uniformly LFP-definable. Then winning regions are uniformly LFP-definable.

In what follows we present a proof of Theorem 12. A detailed description of the model checking games used in the argument can be found in Chapter 3 of [9].

Part (a): Let $\mathcal{P} \mathcal{G}$ be the class of parity games with an unbounded number of priorities.

Lemma 13. There is a formula $\psi(S, w) \in \mathrm{LFP}$ with a free first-order variable $w$ and a free binary second-order variable $S$, that defines those positions $w$ of a parity game $\mathcal{G} \in$ $\mathcal{P G}$, such that on every infinite $S$-path from $w$ the lowest priority seen infinitely often is even.
Proof. Given a binary relation $S$, we let $\varphi(S, w, \mu):=$

$$
\begin{aligned}
& {[\operatorname{gfp} X x \cdot[\operatorname{lfp} Y y \cdot[\operatorname{gfp} Z z} \\
& \left.\left.\left.\qquad\left[\begin{array}{l}
(\Omega(z)<\mu \wedge \forall u(S z u \rightarrow X u)) \vee \\
(\Omega(z)=\mu \wedge \forall u(S z u \rightarrow Y u)) \vee \\
(\mu<\Omega(z) \wedge \forall u(S z u \rightarrow Z u))
\end{array}\right]\right](w)\right](x)\right](y)
\end{aligned}
$$

We claim that this formula defines the set of pairs $(w, \mu)$ such that every infinite $S$-path from $w$ either contains infinitely many nodes $z$ with $\Omega(z)<\mu$, or only finitely many nodes with $\Omega(z)=\mu$.

To see this, consider the model checking game for this formula, played on a graph $(V, S)$ with a fixed node $w$ and a fixed priority $\mu$. Let $\alpha(z)$ be the subformula inside the fixed point definitions. At position $\alpha(z)$ in the model checking game, Player 0 chooses the right disjunct, according to the priority of $z$, and then Player 1 takes the game from $z$ to an $S$-successor $u$ of $z$; via $X u, Y u$ or $Z u$ the play then proceeds to position $\alpha(u)$.

Thus a play in the model checking game essentially amounts to the choice of an $S$-path from $w$ by Player 1 . Such a play is won by Player 0 if the outermost fixed point variable seen infinitely often is either $X$ or $Z$. But this is the case if either a priority smaller than $\mu$ is seen infinitely often or, if this is not the case, priority $\mu$ occurs only finitely often on the path.

Thus, the formula

$$
\psi(S, w):=\forall \mu(\operatorname{Odd}(\mu) \rightarrow \varphi(S, w, \mu))
$$

defines those elements $w$ such that on every infinite $S$-path from $w$ the lowest priority occurring infinitely often is even.

Proof of Theorem 12(a). We assume that there is a formula $\varphi(x, y) \in \mathrm{LFP}$ that defines a complete winning strategy for Player 0 on $\mathcal{K} \subseteq \mathcal{P} \mathcal{G}$. Let $\mathcal{G} \in \mathcal{K}$ be a parity game and let $S$ be the strategy defined by $\varphi$ on $\mathcal{G}$. The closure of $S$ is defined by the formula

$$
\varphi^{*}(x, y):=\varphi(x, y) \vee\left(V_{1} x \wedge E x y\right)
$$

Since $S$ is a winning strategy on $W_{0}$, the lowest priority seen infinitely often on every infinite $\hat{S}$-path that starts from $W_{0}$ is even. Lemma 13 states that there is an LFP-formula $\psi(S, w)$ that defines those positions $w$ of a parity game $\mathcal{G} \in \mathcal{P} \mathcal{G}$ such that on every infinite $S$-path from $w$ the lowest priority seen infinitely often is even. Furthermore, for every position $v \in V_{0}$ in the winning region of Player 0 there is a position $u$ with $(v, u) \in E$ such that $\mathcal{G} \models \varphi(v, u)$, since $\varphi$ defines a winning strategy for Player 0 . Hence, the following LFPformula defines the winning region of Player 0 in $\mathcal{G}$.

$$
\psi^{*}(w):=\psi\left(w, S x y / \varphi^{*}(x, y)\right)
$$

The reasoning for Player 1 is analogous. 
Part (b): Part (b) is proved via an analogue to Lemma 13, using that $\omega$-regular languages are MSO-definable.

Lemma 14. Let $\psi$ be an MSO-formula over infinite words, and let $\mathcal{W}$ be the language described by $\psi$. Then, there exists an LFP-formula $\varphi(S, v)$ with a free first-order variable $v$ and a free binary second-order variable $S$ that defines those positions $v$ in an arena from where the labeling of every infinite $S$-path is contained in $\mathcal{W}$.

Proof. Assume that there is a bisimulation-invariant MSOformula $\tilde{\varphi}(S, v)$ that defines the respective positions. By [12], there exists an equivalent formula $\widetilde{\varphi}^{\prime} \in L_{\mu}$ such that $(\mathcal{A}, S, v)=\widetilde{\varphi}^{\prime}$ if, and only if, the labeling of every infinite $S$-path from $v$ is contained in $\mathcal{W}$. As $L_{\mu} \subseteq \mathrm{LFP}$, the lemma follows.

To see that such a bisimulation-invariant formula $\widetilde{\varphi}(S, v)$ exists, let $\vartheta$ be as follows:

$$
\vartheta:=\forall X(S-\operatorname{Path}(X) \rightarrow \psi(X)),
$$

where $S-\operatorname{Path}(X):=X v \wedge \forall x(X x \rightarrow S x \wedge \exists ! y(E x y \wedge X y))$. It follows that $v$ in $(\mathcal{A}, S)$ has the desired property if the unfolding $\operatorname{Unf}(\mathcal{A}, S, v)=\vartheta$. By [7], there exists a formula $\widetilde{\varphi}(S, v)$ as required, but for the bisimulation-invariance. To see that the $\widetilde{\varphi}$ is indeed bisimulation-invariant, assume the contrary. Then, there exist two bisimilar structures $(\mathcal{A}, S, v) \approx$ $\left(\mathcal{A}^{\prime}, S^{\prime}, v^{\prime}\right)$ with $(\mathcal{A}, S, v) \models \widetilde{\varphi},\left(\mathcal{A}^{\prime}, S^{\prime}, v^{\prime}\right) \not \models \widetilde{\varphi}$. It follows that $\operatorname{Unf}(\mathcal{A}, S, v) \models \vartheta$, and $\operatorname{Unf}\left(\mathcal{A}^{\prime}, S^{\prime}, v^{\prime}\right) \not \models \vartheta$. This is a contradiction, as $\vartheta$ is clearly bisimulation-invariant, and $\operatorname{Unf}(\mathcal{A}, S, v) \approx \operatorname{Unf}\left(\mathcal{A}^{\prime}, S^{\prime}, v^{\prime}\right)$.

As a corollary of Theorem 12 (a), we obtain a nondefinability result for winning strategies on $\mathcal{P G}$ from the corresponding result for winning regions [8, Theorem 9].

Corollary 15. Complete winning strategies on the class $\mathcal{P G}$ are not definable in LFP, even under the assumption that the game graph is countable and the number of priorities is finite.

We proceed to study the converse translation, from winning regions to complete winning strategies: is it true that for LFP, in general, the definability of winning regions implies the definability of complete (positional) winning strategies? At least we were able to obtain such translations for the case of parity games with a bounded number of priorities in Section V. We start by specifying the formal setting.

Definition 16. A class $\mathcal{K}$ of arenas allows LFP-translations for Player $\sigma$ (of LFP-definable winning regions to LFPdefinable positional winning strategies) if for every winning condition $\mathcal{W} \subseteq \omega^{\omega}$ which guarantees positional winning strategies on $\mathcal{K}$ for Player $\sigma$ and for which $(\mathcal{K}, \mathcal{W})$ allows LFP-definable winning regions for Player $\sigma$, the pair $(\mathcal{K}, \mathcal{W})$ also allows LFP-definable (positional) winning strategies.

The main question now reads as follows: does the class $\mathcal{K}_{\text {all }}$ of all game arenas allow LFP-translations? We can give the following partial answers to this question.
Theorem 17. There is a class $\mathcal{K}=\left\{G_{i}: i \in \omega\right\}$ consisting of countably infinite game arenas $G_{i}$ which does not allow LFP-translations.

Proof. We construct a class $\mathcal{K}$ of game arenas which does not allow LFP-translations (for Player 0). The class $\mathcal{K}$ consists of a countable family of game arenas $G_{i}$. We develop a corresponding winning condition $\mathcal{W}$ such that the winning regions in all games $\mathcal{G}_{i}=\left(G_{i}, \mathcal{W}\right)$ are trivial, meaning that Player 0 wins from every position (hence, the pair $(\mathcal{K}, \mathcal{W})$ clearly allows LFP-definable winning regions for Player 0). Moreover, the objective of Player 0 essentially is a parity winning condition, which means that he has positional winning strategies on his winning region. However, while the winning regions in the games $\mathcal{G}_{i}=(G, \mathcal{W})$ are trivial, fixing a positional winning strategy is hard, since this requires to solve a family of parity games with an unbounded set of priorities (which is known to be undefinable in LFP in general). This shows that the pair $(\mathcal{K}, \mathcal{W})$ does not allow LFP-definable winning strategies for Player 0 , which in turn implies that $\mathcal{K}$ does not allow LFP-definable translations.

We proceed to give the formal construction. Let $\left(\varphi_{i}(x)\right)_{i \in \omega}$ be an enumeration of all LFP-formulae with one free variable over the signature of arithmetic. Moreover, let $\mathcal{M}_{i}$ denote the model checking game (with parity winning condition) for $\varphi_{i}(x)$ on $\mathfrak{N}=(\mathbb{N},+, \cdot)$. For every $n \in \mathbb{N}$ there exists a position $v_{n}$ in $\mathcal{M}_{i}$ such that Player 0 wins the parity game $\mathcal{M}_{i}$ from position $v_{n}$ if, and only if, $\mathfrak{N}=\varphi_{i}(n)$. We also consider the dual parity game $\mathcal{M}_{i}^{d}$ which is won by Player 0 from position $v_{n}$ if, and only if, $\mathfrak{N} \not \varphi_{i}(n)$. Without loss of generality we assume that the priority 0 does not occur in any of the games $\mathcal{M}_{i}$ and $\mathcal{M}_{i}^{d}$. We let $\mathcal{K}=\left\{G_{i}: i \in \omega\right\}$ where the game arena $G_{i}$ is depicted in Figure 1 and we set

$$
\mathcal{W}=\left\{\pi \in[\omega]^{\omega}: \text { if } 0 \in \Omega(\pi), \text { then } \min (\inf (\pi)) \text { is even }\right\}
$$

Hence, Player 0 either has to avoid the special priority 0 or he has to satisfy the parity winning condition. Consequently, Player 0 wins every play in the game $\mathcal{G}_{i}=\left(G_{i}, \mathcal{W}\right)$ which does not start at position $\varphi_{i}(x)$ or at position $\varphi_{i}(n)$ for $n \in \mathbb{N}$. In a play which starts at position $\varphi_{i}(x)$, Player 1 first chooses a natural number $n \in \mathbb{N}$ and Player 0 has to decide at the following position $\varphi_{i}(n)$ whether he wants to play the model checking game $\mathcal{M}_{i}$ from position $v_{n}$ (which he wins if $\mathfrak{N} \models$ $\varphi_{i}(n)$ ) or the dual game $\mathcal{M}_{i}^{d}$ from the same position (which he wins if $\left.\mathfrak{N} \not \models \varphi_{i}(n)\right)$. Hence, Player 0 wins the game $\mathcal{G}_{i}=$ $\left(G_{i}, \mathcal{W}\right)$ from every position with a positional strategy and thus the pair $(\mathcal{K}, \mathcal{W})$ clearly allows LFP-definable winning regions for Player 0 .

We claim that $(\mathcal{K}, \mathcal{W})$ does not allow LFP-definable winning strategies for Player 0 . Otherwise, assume that some LFP-formula strat $(x, y)$ defines in every game arena $G_{i}$ a complete winning strategy for Player 0 in the game $\mathcal{G}_{i}=$ $\left(G_{i}, \mathcal{W}\right)$. Every arena $G_{i}$ can be defined in $\mathfrak{N}$ using a first-order interpretation. Using this first-order interpretation and the formula $\operatorname{strat}(x, y)$, it is easy to obtain an LFPformula $\varphi_{i}^{*}(x)$ which is equivalent to $\varphi_{i}(x)$ over $\mathfrak{N}$ and which 


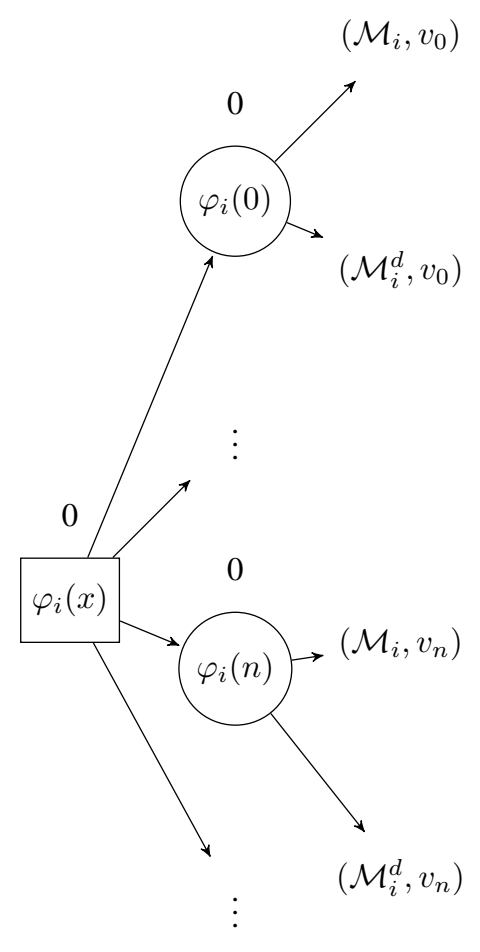

Fig. 1. Game arena $G_{i}$

has the same alternation depth of fixed-point operators as $\operatorname{strat}(x, y)$. This, however, contradicts the strictness of the alternation hierarchy of LFP over $\mathfrak{N}$.

The picture is different when we restrict ourselves to the class $\mathcal{K}_{\text {fin }}$ of all finite game arenas.

Theorem 18. The class $\mathcal{K}_{\text {fin }}$ allows LFP-translations.

To prove this theorem we recall the construction of Otto [20] to show that LFP captures polynomial time on classes of (finite) graphs where all vertices can pairwise be distinguished by the notion of bisimulation.

Given two arenas $G=\left(V \uplus \omega, V_{0}, V_{1}, E, \Omega,<\right)$ and $H=$ $\left(V^{\prime} \uplus \omega, V_{0}^{\prime}, V_{1}^{\prime}, E^{\prime}, \Omega^{\prime},<\right)$ we say that a relation $Z \subseteq V \times V^{\prime}$ is a bisimulation if for all pairs $(x, y) \in Z$ the following conditions are satisfied:

(a) $x \in V_{\sigma}$ if, and only if, $y \in V_{\sigma}^{\prime}$ for $\sigma \in\{0,1\}$ and $\Omega(x)=$ $\Omega^{\prime}(y)$, and

(b) for $\left(x, x^{\prime}\right) \in E$ there exists $\left(y, y^{\prime}\right) \in E^{\prime}$ with $\left(x^{\prime}, y^{\prime}\right) \in Z$, and

(c) for $\left(y, y^{\prime}\right) \in E^{\prime}$ there exists $\left(x, x^{\prime}\right) \in E$ with $\left(x^{\prime}, y^{\prime}\right) \in Z$.

For $v \in V$ and $w \in V^{\prime}$ we write $G, v \sim H, w$ if there exists a bisimulation $Z \subseteq V \times V^{\prime}$ with $(v, w) \in Z$. For the case $G=H$, the maximal bisimulation relation $Z \subseteq V^{2}$ is an equivalence relation on $V$. For what follows, we consider finite game arenas $G$ (which means that $V$ is finite). We construct an IFP-formula $x \prec y$ such that for the formula $x \sim y:=$ $\neg x \prec y \wedge \neg y \prec x$ and every finite game arena $G$ we have: (i) $\left\{(v, w) \in V^{2}: G \models v \sim w\right\}$ is the maximal bisimulation in $G^{+}$, and

(ii) $\left\{(v, w) \in V^{2}: G \mid v \prec w\right\}$ is a strict (linear) preorder on $V$, which means that $\prec$ is a linear order on the set of bisimulation equivalence classes $V^{\sim}=\{[v]: v \in V\}$.

It is easy to see that the following formula has the desired properties $x \prec y:=$

$$
\begin{aligned}
& {\left[\text { ifp } x \prec y:\left(V_{0} x \wedge V_{1} y\right) \vee\left(V_{0} x \leftrightarrow V_{0} y \wedge \Omega(x)<\Omega(y)\right) \vee\right.} \\
& \left(x \sim y \wedge \exists z \left(E x z \wedge \forall z ^ { \prime } ( E y z ^ { \prime } \rightarrow z ^ { \prime } \nsim z ) \wedge \forall z ^ { \prime } \left(z^{\prime} \prec z \rightarrow\right.\right.\right. \\
& \left.\exists z^{\prime \prime}\left(z^{\prime \prime} \sim z^{\prime} \wedge E x z^{\prime \prime}\right) \leftrightarrow \exists z^{\prime \prime}\left(z^{\prime \prime} \sim z^{\prime} \wedge E y z^{\prime \prime}\right)\right](x, y) .
\end{aligned}
$$

Since LFP $=$ IFP we know that the strict preorder $\prec$ can also be defined by an LFP-formula. The bisimulation quotient $[G]$ of $G$ results from taking the quotient with respect to bisimulation, i.e. we have $[G]=\left(V^{\sim} \uplus \omega, V_{0}^{\sim}, V_{1}^{\sim}, E^{\sim}, \Omega^{\sim},<\right)$ where

- $V^{\sim}=\{[v]: v \in V\}$ and $V_{\sigma}^{\sim}=\left\{[v]: v \in V_{\sigma}\right\}$, and

- $E^{\sim}=\{([v],[w]):([v] \times[w]) \cap E \neq \emptyset\}$, and $\Omega^{\sim}([v])=$ $\Omega(v)$.

The LFP-definable preorder $\prec$ yields an LFP-definable linear order on $V^{\sim}$. The importance of the notion of bisimulation stems from the fact that winning regions are preserved. Indeed this holds for arbitrary winning conditions.

Lemma 19. Let $\mathcal{W} \subseteq \omega^{\omega}$ be a winning condition and let $G=$ $\left(V \uplus \omega, V_{0}, V_{1}, E, \Omega,<\right)$ and $H=\left(V^{\prime} \uplus \omega, V_{0}^{\prime}, V_{1}^{\prime}, E^{\prime}, \Omega^{\prime},<\right)$ be two arenas with $v \in V$ and $w \in V^{\prime}$. If $G, v \sim H$, w then Player $\sigma$ has a winning strategy in $(G, \mathcal{W})$ from $v$ if, and only if, she has a winning strategy from $w$ in $(H, \mathcal{W})$.

Proof. Let $g: V^{\star} V_{\sigma} \rightarrow V$ be a winning strategy of Player $\sigma$ from $v \in V$ in $(G, \mathcal{W})$. We describe a winning strategy for Player $\sigma$ starting from $w$ in $(H, \mathcal{W})$. During a play $\pi_{H}=w_{0} w_{1} w_{2} \cdots \in V^{\prime \omega}$ starting from $w_{0}=w$ in $(H, \mathcal{W})$ he ensures that there is a corresponding play $\pi_{G}=v_{0} v_{1} v_{2} \cdots \in$ $V^{\omega}$ starting from $v_{0}=v$ in $(G, \mathcal{W})$ which is consistent with the strategy $g$ such that $G, v_{i} \sim H, w_{i}$. For the inductive step we consider two cases. If $w_{i} \in V_{1-\sigma}^{\prime}$ then the next position $w_{i+1} \in V^{\prime}$ with $\left(w_{i}, w_{i+1}\right) \in F$ is chosen by Player $1-\sigma$. Since $G, v_{i} \sim H, w_{i}$ we have that $v_{i} \in V_{1-\sigma}$ and there exists $v_{i+1} \in V$ such that $\left(v_{i}, v_{i+1}\right) \in E$ and $G, v_{i+1} \sim H, w_{i+1}$. Moreover, the extension of the partial play $v_{0} v_{1} \cdots v_{i}$ to $v_{0} v_{1} \cdots v_{i} v_{i+1}$ yields a partial play in $(G, \mathcal{W})$ which is still consistent with the winning strategy $g$.

If $w_{i} \in V_{\sigma}^{\prime}$ then also $v_{i} \in V_{\sigma}$. We choose $v_{i+1}=$ $g\left(v_{0} \cdots v_{i}\right)$. Then $\left(v_{i}, v_{i+1}\right) \in E$ and $v_{0} \cdots v_{i} v_{i+1}$ is a partial play in $(G, \mathcal{W})$ which is still consistent with $g$. Since $G, v_{i} \sim$ $H, w_{i}$ we can find $w_{i+1} \in V^{\prime}$ such that $\left(w_{i}, w_{i+1}\right) \in E^{\prime}$ and $G, v_{i+1} \sim H, w_{i+1}$ (and this is the choice of Player $\sigma$ at position $w_{0} \cdots w_{i}$ ).

Assume that Player $\sigma$ plays according to this strategy and produces a play $\pi_{H}=w_{0} w_{1} \cdots$ in $(H, \mathcal{W})$ together with the corresponding play $\pi_{G}=v_{0} v_{1} \cdots$ in $(G, \mathcal{W})$. Since $\pi_{G}$ is consistent with $g$ we know that $\Omega\left(\pi_{G}\right) \in \mathcal{W}_{\sigma}$. However, since $G, v_{i} \sim H, w_{i}$ we have $\Omega\left(v_{i}\right)=\Omega^{\prime}\left(w_{i}\right)$ which means that 
$\Omega\left(\pi_{G}\right)=\Omega^{\prime}\left(\pi_{H}\right)$. Hence $\Omega^{\prime}\left(\pi_{H}\right) \in \mathcal{W}_{\sigma}$ and Player $\sigma$ wins $\pi_{H}$ as claimed.

In particular we have $G, v \sim[G],[v]$. Hence, if $W_{\sigma}$ denotes the winning region of Player $\sigma$ in $(G, \mathcal{W})$ and $W_{\sigma}^{\sim}$ denotes the winning region of Player $\sigma$ in $([G], \mathcal{W})$ we have $W_{\sigma}^{\sim}=\{[v]$ : $\left.v \in W_{\sigma}\right\}$ and $W_{\sigma}=\bigcup W_{\sigma}^{\sim}$. Moreover, winning strategies of Player $\sigma$ in $[G]$ can easily be lifted to winning strategies for Player $\sigma$ in $G$.

Lemma 20. Let $S^{\sim} \subseteq V_{\sigma}^{\sim} \times V^{\sim}$ be a complete positional winning strategy for Player $\sigma$ in $([G], \mathcal{W})$ on her winning region $W_{\sigma}^{\sim}$. Then $S=\left\{(v, w) \in V_{\sigma} \times V:([v],[w]) \in\right.$ $\left.S^{\sim},(v, w) \in E\right\}$ is a complete positional winning strategy for Player $\sigma$ on her winning region $W_{\sigma}$ in $(G, \mathcal{W})$.

Proof. First we observe that for every $v \in V_{\sigma}$, the strategy $S$ contains at least one pair $(v, w)$ with $(v, w) \in E$. Indeed, for $[v] \in V_{\sigma}^{\sim}$, the strategy $S^{\sim}$ contains a pair $([v],[w]) \in S^{\sim}$ with $([v],[w]) \in E^{\sim}$, hence for some $w^{\prime} \in[w]$ we have that $\left(v, w^{\prime}\right) \in S$.

Let $\pi=v_{0} v_{1} v_{2} v_{3} \cdots$ be a play in $(G, \mathcal{W})$ starting from a vertex $v_{0} \in W_{\sigma}$ which is consistent with $S$. As we observed above we have that $\left[v_{0}\right] \in W_{\sigma}^{\sim}$. We claim that $[\pi]=\left[v_{0}\right]\left[v_{1}\right]\left[v_{2}\right] \cdots$ is a play in $[G]$ which is consistent with $S^{\sim}$.

For the case where $v_{i} \in V_{\sigma}$ we have that $\left(\left[v_{i}\right],\left[v_{i+1}\right]\right) \in$ $S^{\sim}$ which already shows that $\left[v_{0}\right]\left[v_{1}\right] \cdots\left[v_{i}\right]\left[v_{i+1}\right]$ is a partial play which is consistent with $S^{\sim}$. If $v_{i} \in V_{1-\sigma}$ we have that $\left(v_{i}, v_{i+1}\right) \in E$ and $\left[v_{i}\right] \in V_{1-\sigma}^{\sim}$, thus $\left(\left[v_{i}\right],\left[v_{i+1}\right]\right) \in E^{\sim}$ and again $\left[v_{0}\right]\left[v_{1}\right] \cdots\left[v_{i}\right]\left[v_{i+1}\right]$ still is a partial play which is consistent with $S^{\sim}$.

Since $[\pi]$ is a play in $([G], \mathcal{W})$ which starts at vertex $\left[v_{0}\right] \in$ $W_{\sigma}^{\sim}$ and which is consistent with $S^{\sim}$ we know that $\Omega^{\sim}([\pi]) \in$ $\mathcal{W}_{\sigma}$. Since $\Omega\left(v_{i}\right)=\Omega^{\sim}\left(\left[v_{i}\right]\right)$ we conclude that $\Omega(\pi) \in \mathcal{W}_{\sigma}$ which shows that Player $\sigma$ wins the play.

To prove Theorem 18 it thus suffices to construct an LFPformula $\varphi(x, y)$ which defines a positional winning strategy for Player $\sigma$ on the bisimulation quotient $[G]$. Since there exists a formula $\varphi(x)$ which defines the winning region $W_{\sigma}^{\sim}$ of Player $\sigma$ in $[G]$ we can assume that $V^{\sim}=W_{\sigma}^{\sim}$.

First, we observe that the linear order $\prec$ on $V^{\sim}$ also induces a linear order on $E^{\sim}$. Hence, the following inductive procedure can easily be expressed in LFP, since on ordered structures LFP can express all polynomial-time computable functions (see e.g. [9]). Initially we let $S^{\sim}=\emptyset$ and $H=[G]$. We then choose the minimal position $[v] \in V_{\sigma}^{\sim}$ for which no outgoing edge has been selected so far, i.e. for which $S^{\sim} \cap\left\{([v],[w]) \in E^{\sim}\right\}=\emptyset$. Let $([v],[w]) \in E^{\sim}$ be the minimal edge such that Player $\sigma$ still wins from $[v]$ in the modified game $H\{([v],[w])\}$ which arises from $H$ by deleting all other outgoing edges at position $[v]$ except for $([v],[w])$. We let $S^{\sim}:=S^{\sim} \cup\{([v],[w])\}$ and $H:=H\{([v],[w])\}$ and continue this inductive process until all vertices in $V_{\sigma}^{\sim}$ have been processed. In this way we obtain a positional winning strategy for Player $\sigma$ in $[G]$.
Theorem 18 has an interesting implication on the LFPdefinability of winning strategies in parity games over finite game arenas. It was shown in [8] that, over finite game arenas, winning regions in parity games are LFP-definable if, and only if, they are computable in polynomial time. By Theorem 18 this also holds for winning strategies.

Corollary 21. On the class of all finite game arenas, winning strategies in parity games are definable in LFP if, and only if, they are computable in polynomial time.

\section{REFERENCES}

[1] D. Berwanger. Game logic is strong enough for parity games. Studia Logica, 75(2):205-219, 2003.

[2] D. Berwanger, A. Dawar, P. Hunter, and S. Kreutzer. Dag-width and parity games. In Proceedings of 23rd Annual Symposium on Theoretical Aspects of Computer Science, STACS 2006, Lecture Notes in Computer Science Nr. 3848, pages 524-536, 2006.

[3] D. Berwanger and E. Grädel. Fixed-point logics and solitaire games. Theory of Computing Systems, 37:675-694, 2004

[4] D. Berwanger, E. Grädel, Ł. Kaiser, and R. Rabinovich. Entanglement and the Complexity of Directed Graphs. Theoretical Computer Science, 463(0):2-25, 2012. Special Issue on Theory and Applications of Graph Searching Problems.

[5] D. Berwanger, E. Grädel, and G. Lenzi. The variable hierarchy of the $\mu$-calculus is strict. Theory of Computing Systems, 40:437-466, 2007.

[6] A. Blumensath and E. Grädel. Finite presentations of infinite structures: Automata and interpretations. Theory of Computing Systems, 37:641 674, 2004.

[7] B. Courcelle and I. Walukiewicz. Monadic second-order logic, graph coverings and unfoldings of transition systems. Annals of Pure and Applied Logic, 92:35-62, 1998.

[8] A. Dawar and E. Grädel. The Descriptive Complexity of Parity Games. In Proceedings of the 17th Annual Conference on Computer Science Logic, CSL 2008, volume 5213 of LNCS, pages 354-368. Springer, 2008.

[9] E. Grädel et al. Finite Model Theory and Its Applications. SpringerVerlag, 2007.

[10] Y. Gurevich and S. Shelah. Fixed-point extensions of first-order logic. Annals of Pure and Applied Logic, 32:265-280, 1986.

[11] P. Hunter. Complexity and Infinite Games on Finite Graphs. $\mathrm{PhD}$ thesis, University of Cambridge, 2007.

[12] D. Janin and I. Walukiewicz. On the expressive completeness of the propositional mu-calculus with respect to monadic second order logic. In Proceedings of 7th International Conference on Concurrency Theory CONCUR '96, number 1119 in Lecture Notes in Computer Science, pages 263-277. Springer-Verlag, 1996.

[13] M. Jurdziński. Small progress measures for solving parity games. In Proceedings of 17th Annual Symposium on Theoretical Aspects of Computer Science, STACS 2000, Lecture Notes in Computer Science Nr. 1770, pages 290-301. Springer, 2000.

[14] M. Jurdziński, M. Paterson, and U. Zwick. A deterministic subexponential algorithm for solving parity games. In Proceedings of ACM-SIAM Proceedings on Discrete Algorithms, SODA 2006, pages 117-123, 2006.

[15] S. Kreutzer. Expressive equivalence of least and inflationary fixed-point logic. Annals of Pure and Applied Logic, 130(1-3):61-78, 2004.

[16] Y. Moschovakis. Elementary induction on abstract structures. North Holland, 1974.

[17] J. Obdržálek. Fast mu-calculus model checking when tree-width is bounded. In Proceedings of CAV 2003, volume 2752 of LNCS, pages 80-92. Springer, 2003.

[18] J. Obdržálek. DAG-width - connectivity measure for directed graphs. In Proceedings of ACM-SIAM Proceedings on Discrete Algorithms, SODA 2006, pages 814-821, 2006

[19] J. Obdržálek. Clique-width and parity games. In Computer Science Logic, 21st International Workshop, CSL 2007, volume 4646 of Lecture Notes in Computer Science, pages 54-68. Springer, 2007.

[20] M. Otto. Bisimulation-invariant Ptime and higher-dimensional mucalculus. Theoretical Computer Science, 224:237-265, 1999.

[21] I. Walukiewicz. Monadic second-order logic on tree-like structures. Theoretical Computer Science, 275:311-346, 2001. 